Tropical Journal of Pharmaceutical Research January 2021; 20 (1): 53-60

ISSN: $1596-5996$ (print); 1596-9827 (electronic)

(C) Pharmacotherapy Group, Faculty of Pharmacy, University of Benin, Benin City, 300001 Nigeria.

\title{
Serum Sestrin2 and PIGF levels in pregnant women with pre-eclampsia and their correlations with the severity of the disease: A case-control study
}

\author{
Lijun Li, Zhen Sun, Shasha Liu, Hongyan Cui \\ Obstetrics Department, Tianjin Central Hospital of Gynecology Obstetrics, 156 Nankai 3rd Road, Nankai, Tianjin, 300100, \\ China \\ *For correspondence: Email: yhf56685@126.com
}

Sent for review: 28 September 2019

Revised accepted: 17 December 2020

\begin{abstract}
Purpose: Sestrin2, a metabolic regulator with antioxidant activity, might have some certain predictability for the occurrence and severity of pre-eclampsia (PE). The purpose of this work was to explore the levels of serum Sestrin2 and PIGF in pregnant women with PE and their correlation with the severity index of the disease.

Methods:This was a retrospective case-control study of pregnant women with PE who planned to give birth in our hospital from 05/2017 to 05/2019. Pearson correlation was used to analyze Sestrin2 and PIGF levels with PE severity. Receiver operating characteristic (ROC) curves were used to evaluate the predictive value of Sestrin2 and PIGF for PE.

Results: There were 52 women in the control group, 46 in the mild PE group, and 36 in the severe $P E$ group. As the severity of PE increased, the median levels of Sestrin2 increased (8.1, 9.8, and 11.8 $\mathrm{ng} / \mathrm{ml})$, and the levels of PIGF decreased $(156,117$, and $67 \mathrm{pg} / \mathrm{ml}$ ) in the three groups of women (all $P<0.05)$. The levels of Sestrin2 and PIGF were strongly correlated with mean arterial pressure, proteinuria, newborn birth weight and gestational weeks at delivery (all $P<0.001$ ). Sestrin2 and PIGF had high efficiency of diagnosing PE (cut-off: $8.90 \mathrm{ng} / \mathrm{ml}$, Area Under Curve [AUC]=0.979; cut-off: $122.50 \mathrm{pg} / \mathrm{ml}$, AUC=0.963). Additionally, Sestrin2 and PIGF showed high value of severity prediction (cut-off: $11.15 \mathrm{ng} / \mathrm{ml}$, AUC=0.857; cut-off: $68.50 \mathrm{pg} / \mathrm{ml}$, AUC=0.837).

Conclusion: Sestrin2 and PIGF are correlated with the severity of PE. Both Sestrin2 and PIGF had high value for PE diagnosis and severity prediction.
\end{abstract}

Keywords: pre-eclampsia; Sestrin2; placental growth factor; oxidative stress; severity

\begin{abstract}
This is an Open Access article that uses a fund-ing model which does not charge readers or their institutions for access and distributed under the terms of the Creative Commons Attribution License (http://creativecommons.org/licenses/by/4.0) and the Budapest Open Access Initiative (http://www.budapestopenaccessinitiative.org/read), which permit unrestricted use, distribution, and reproduction in any medium, provided the original work is properly credited.
\end{abstract}

Tropical Journal of Pharmaceutical Research is indexed by Science Citation Index (SciSearch), Scopus, International Pharmaceutical Abstract, Chemical Abstracts, Embase, Index Copernicus, EBSCO, African Index Medicus, JournalSeek, Journal Citation Reports/Science Edition, Directory of Open Access Journals (DOAJ), African Journal Online, Bioline International, Open-J-Gate and Pharmacy Abstracts

\section{INTRODUCTION}

Hypertensive disorder during pregnancy seriously threatens the health of pregnant women and newborns. About $2 \%-10 \%$ of pregnancies in the world are affected by it [1-5].
The incidence of pre-eclampsia (PE) is higher among women with hypertensive disorders, for a prevalence of about $3 \%$ among pregnant women [2-5]. The severity and onset of PE are closely related to the prognosis of pregnant women. Currently, the pathogenesis of PE is not fully 
understood, but the possible causes include abnormal placental implantation, angiogenic factors, cardiovascular maladaptation and vasoconstriction, genetic predisposition, immunelogic phenomena, and vascular endothelial damage, among others [2, 3].

$P E$ results in reduced organ perfusion due to vasospasms and activation of the coagulation cascade [6], leading to early placental ischemia and hypoxia [2, 3]. Late placental ischemiareperfusion injury leads to a placental oxidative stress reaction and the release of various inflammatory factors into circulation, triggering a systemic inflammatory reaction and maternal diseases [7]. The placental growth factor (PIGF) can promote vascular regeneration and improve blood circulation [8-10]. As a member of the Sesn family, Sestrin2 is a metabolic regulator, and its expression increases in the presence of DNA damage, hypoxia, oxidative stress, and other adverse metabolic conditions. Sestrin2 has antioxidant activity [11, 12], suggesting that it might have some certain predictability for the occurrence and severity of PE $[13,14]$.

At present, there are many studies on the relation of PE with PIGF [15-17], but there are few regarding the association of Sestrin2. Therefore, the aim of this study was to explore the levels of serum Sestrin2 and PIGF in pregnant women with $\mathrm{PE}$ and their correlation with the severity index of the disease. The results could provide a novel biomarker for the prediction, diagnosis, and management of PE.

\section{METHODS}

\section{Study design and patients}

This was a retrospective case-control study of pregnant women with PE who planned to give birth in our hospital between May 2017 and May 2019. This study was approved by the Academic Ethics Committee of the Tianjin Central Hospital of Gynecology Obstetrics, China.

The inclusion criteria were: 1) met the diagnostic criteria of PE and severe PE according to the Guidelines for the Diagnosis and Treatment of Hypertensive Disorder Complicating Pregnancy (2015) issued by the Chinese Medical Association [18]; 2) pre-pregnancy blood pressure <140/90 mmHg; 3) >20 weeks of gestation; 4) single-fetus pregnancy and live birth; 5) mother of 20-40 years of age; and 6) no history of hypertension treatment. The exclusion criteria were: 1) history of chronic diseases such as hypertension, heart disease, diabetes, nephropathy, and autoimmune diseases; 2) history of proteinuria occurring before 20 weeks of pregnancy; 3) multiple gestation pregnancy; 4) premature rupture of membranes; or 5) fetal malformation.

Women with a normal pregnancy matched for age, gestational age, and body mass index (BMI) with the women with $\mathrm{PE}$ during the same period were selected as the control group.

\section{Diagnostic criteria}

The diagnostic criteria for PE were: 1) systolic blood pressure $\geq 140$ and/or diastolic blood pressure $\geq 90 \mathrm{mmHg}$ after 20 weeks of pregnancy; and 2) accompanied by urinary protein $\geq 0.3 \mathrm{~g} / 24 \mathrm{~h}$, or urinary protein/creatinine $\geq 0.3$, or random urinary protein + or above; or abnormal changes in heart, liver, lung, kidney, other important organs, or blood.

The diagnostic criteria for severe PE were: 1) systolic blood pressure $\geq 160 \mathrm{mmHg}$ and/or diastolic blood pressure $\geq 110 \mathrm{mmHg}$; 2) central nervous system symptoms such as visual impairment or headache; 3) persistent upper abdominal pain; 4) abnormal elevation of liver enzymes; 5) progressive renal function impairment: urinary protein $>2.0 \mathrm{~g} / 24 \mathrm{~h}$; oliguria or serum creatinine $(\mathrm{sCr})>106 \mu \mathrm{mol} / \mathrm{L} ; 6)$ heart failure or pulmonary edema; 7) ascites, pleural effusion, or pericardial effusion; 8) platelets decreased continuously and less than $100 \times 10^{9} / \mathrm{L}$; 9) microvascular hemolysis; and 10) fetal growth restriction or placental abruption, fetal death in utero, etc.

Patients who met the diagnostic criteria for PE but not severe PE were divided into mild PE group.

\section{Management}

Oral antihypertensive drugs were given, mainly labetalol 50-100 mg, 3 times/day, or nifedipine 10 mg, 3 times/day. If necessary, intravenous nicardipine was given at $\geq 1 \mathrm{mg} / \mathrm{h}$, and the dose was adjusted according to blood pressure. The first choice for spasmolysis was intravenous magnesium sulfate, with a loading dose of $20 \mathrm{ml}$ of $25 \%$ magnesium sulfate dissolved in $100 \mathrm{ml}$ of $5 \%$ glucose over $15-20 \mathrm{~min}$, with a maintenance dose of $1-2 \mathrm{~g} / \mathrm{h}$. The total amount of magnesium sulfate was no more than $25 \mathrm{~g} /$ day, administered for 6-12 $\mathrm{h}$ per day and less than 5 days. For sedation, oral diazepam 2.5-5 $\mathrm{mg}$ or $10 \mathrm{mg}$ intramuscular was given before going to bed. For diuresis, intravenous furosemide was given in case of edema, heart failure, and renal insufficiency. Albumin was supplemented before diuretics in case of hypoproteinemia, and mannitol was given in case of cerebral edema. Pregnant women with $<34$ weeks of gestation and who were expected to give birth within 1 week were given glucocorticoids (dexamethasone $6 \mathrm{mg}$ for intramuscular injection, once 
every $12 \mathrm{~h}$, four injections) to promote fetal lung maturation. According to the patients' conditions, gestational weeks, and fetal conditions, the pregnancy was terminated in due course.

\section{Data collection}

All pregnant women routinely underwent an obstetric examination, including blood pressure, body weight, uterine height, abdominal circumference, fetal heart rate, fetal position, and obstetric ultrasound to evaluate fetal growth. The patients with PE had a weekly prenatal check-up after diagnosis. ECG, fetal heart monitoring, blood biochemistry (including liver function, renal function, blood sugar, blood lipid, electrolyte, and myocardial enzyme), coagulation function, 24-h urine proteins, ultrasound examination of the fetus, placenta, and amniotic fluid were carried out routinely. Color Doppler ultrasound fetal blood flow monitoring was conducted every two weeks. The patients were guided to monitor their blood pressure in the morning, at noon, in the afternoon, and before going to bed. Patients with severe PE were hospitalized for management. In severe PE, fetal heart monitoring was conducted at least once a day, ultrasound examination of the fetus, placenta, and amniotic fluid, and color Doppler ultrasound fetal blood flow monitoring were carried out every 5-7 days.

For measuring blood pressure, before measurement, the patient rested quietly for at least $5 \mathrm{~min}$, sitting or lying, limbs relaxed, and the cuff at the same level as the heart. Usually, the right upper limb was used, and both arms were used if necessary. At the first occurrence of hypertension, retests were carried out at intervals of $4 \mathrm{~h}$ or more, and the diagnosis was only made if both times reach the criterion for hypertension. For severe hypertension, i.e., systolic blood pressure $\geq 160 \mathrm{mmHg}$ and/or diastolic blood pressure $\geq 110 \mathrm{mmHg}$, the patients could be diagnosed by repeated measurement over several minutes.

Urine was collected for $24 \mathrm{~h}$ urine for 24-h urine protein examination. For patients in mild PE and severe PE group, $5 \mathrm{ml}$ of venous blood was drawn and stored in EDTA at the diagnosis of $\mathrm{PE}$, while patients in control group were sampling at matching gestational week of 30-37 weeks. After centrifugation at $4^{\circ} \mathrm{C}$ and $3000 \mathrm{rpm}$ for 15 $\min$, serum was taken and stored at $-80^{\circ} \mathrm{C}$. Serum Sestrin2 and PIGF levels were determined by ELISA.

\section{Statistical analysis}

Data processing was performed using SPSS 22.0 (IBM, Armonk, NY, USA). Continuous data were expressed as means \pm standard deviations or median (range) based on the Kolmogorov-
Smirnov test of normal distribution. ANOVA or Kruskall-Wallis test was performed with the Bonferroni or Dunnett post hoc test to examine the group differences. Spearman correlation analysis was used to analyze the correlation between Sestrin2, PIGF and PE severity. The difference in serum sestrin2 and PLGF levels among the three groups of patients is shown by box plots. Receiver operating characteristic (ROC) curves were used to evaluate the predictive value of Sestrin2 and PIGF for PE (control vs. PE, irrespective of severity) and PE severity (mild vs. severe PE). The area under the curve (AUC) was calculated, as well as sensitivity and specificity. Two-sided P-values $\leq 0.05$ were considered statistically significant.

\section{RESULTS}

\section{Characteristics of the patients}

A total of 134 pregnant women were included: 52 cases in the control group, 46 in the mild PE group, and 36 in the severe PE group. There were no significant differences in age, gestational age at blood sampling, BMI, and platelets among the three groups (all $P>0.05$ ) (Table 1). The mean arterial pressure (MAP) at blood sampling, $\mathrm{sCr}$, alanine transaminase (ALT), aspartate transaminase (AST), lactate dehydrogenase (LDH), and proteinuria of pregnant women with mild $P E$ were significantly higher than those in the control group (all $P<0.05$ ), and those indexes in the severe PE group were significantly higher than those in the mild PE group; newborn birth weight, gestational age at delivery, and duration between blood sampling and delivery were smaller when comparing mild PE and control group, as well as severe PE and mild PE group (all $\mathrm{P}<0.05)$ (Table 1).

\section{Serum Sestrin2 and PIGF levels}

The median levels of Sestrin2 in the control, mild $\mathrm{PE}$, and severe PE groups were 8.1 (7.0, 9.5), $9.8(8.0,11.4)$, and $11.8(9.5,14.9) \mathrm{ng} / \mathrm{ml}$, while the median levels of PIGF were $156(123,198)$, $117(70,156)$, and $67(36,148) \mathrm{pg} / \mathrm{ml}$, respectively. Patients in the severe $P E$ group had significantly higher levels of Sestrin2 and lower levels of PIGF than those in the control and mild $P E$ groups (all $P<0.05$ ) (Figure 1).

\section{Correlations between PE severity and Sestrin2 and PIGF levels}

The levels of Sestrin2 were strongly and positively correlated with MAP at blood sampling $(r=0.875, \quad P<0.001)$ and proteinuria $(r=0.908$, $\mathrm{P}<0.001)$, while strongly and negatively correlated with newborn birth weight $(r=-0.813$, $P<0.001)$ and gestational weeks at delivery ( $r=-$ 0.869, $\mathrm{P}<0.001$ ) (Table 2). 
Table 1: Comparison of the characteristics of pregnant women

Characteristics $\mathrm{CG}$
$(\mathrm{n}=52)$ $(\mathrm{n}=52)$
$30(23,36)$
$33.7(30.3 .36 .7)$
28.55
$80.58(20.31 .90$

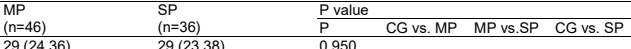
Age (years)
Gestational weeks at blood sampling (weeks) BMM at blood sampling (kg/m)
MAP at blood sampling (mmHG)

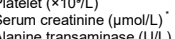

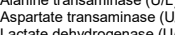

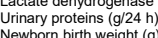
estational weeks at delivery (weeks)

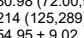
$54.95 \pm 9.02$
$20(8 ., 36)$ $25(6,39)$
$199.7(99.5,230.5$ $3300(2800,3900$
$39.3(37.3,41.4)$

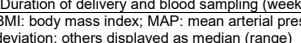

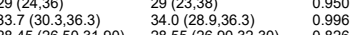

$110.2(97.50,120.50) \quad 128.40(128.50,50,148.20)<0.001<0.001<0.001<<0.001$

(19.2.

$219.9(156.9,250.2)$
$1.51(0.32,1.99)$

$47(19,267)$
$529.0(245.9,783.2)$

$\begin{array}{llll}<0.001 & 0.003 & <0.001 & <0.001\end{array}$

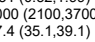

$3325(1500,3200)$
$6.1(32.1,38.1)$

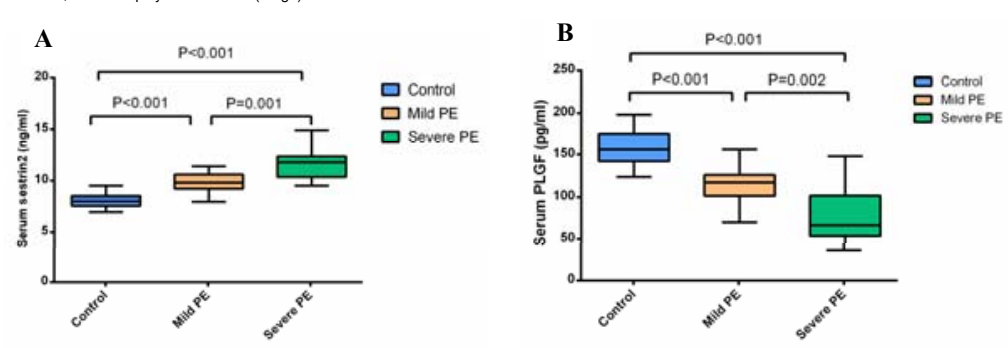

Figure 1: Comparison of serum Sestrin2 (A) and placental growth factor (PIGF) (B) levels in pregnant women

Trop J Pharm Res, January 2021; 20(1): 56 


\begin{tabular}{|c|c|c|c|c|}
\hline \multirow{2}{*}{ Indicator } & \multicolumn{2}{|c|}{ Sestrin2 } & \multicolumn{2}{|c|}{ PLGF } \\
\hline & $\mathrm{r}$ & $P$ & $\mathrm{r}$ & $P$ \\
\hline 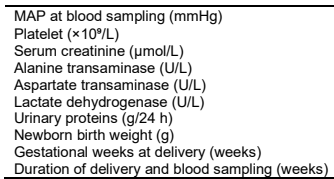 & $\begin{array}{l}0.875 \\
-0.302 \\
0.755 \\
0.564 \\
0.663 \\
0.738 \\
0.098 \\
-0.813 \\
-0.869 \\
-0.682\end{array}$ & $\begin{array}{l}<0.001 \\
<<.001 \\
<<.001 \\
<<.001 \\
<<.001 \\
<<.001 \\
<<.001 \\
<0.001 \\
<<.001 \\
<0.001\end{array}$ & $\begin{array}{l}-0.850 \\
0.306 \\
-0.691 \\
-0.595 \\
-0.655 \\
-0.690 \\
-0.879 \\
0.853 \\
0.900 \\
0.677\end{array}$ & $\begin{array}{l}<0.001 \\
<<.001 \\
<<.001 \\
<<.001 \\
<<.001 \\
<<.001 \\
<<.001 \\
<0.001 \\
<<.001 \\
<0.001\end{array}$ \\
\hline
\end{tabular}

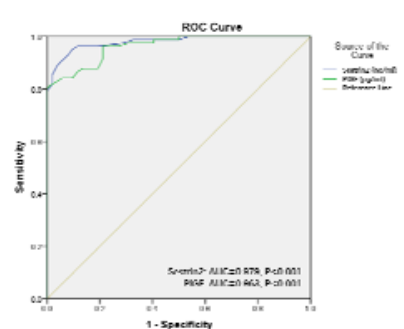

Figure 2: Receiver operating characteristic (ROC) curve of pre-eclampsia predicted
by Sestrin2 and placental growh

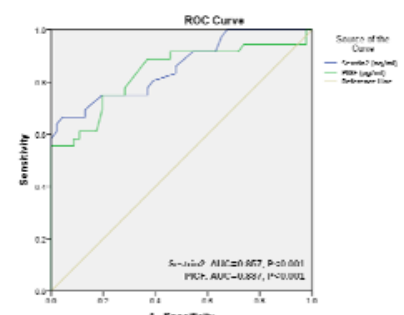

Figure 3: Receiver operating characteristic (ROC) curve of milid and severe pre-
eclampsia predicted by Sestrin2 and placental growhth factor (PIGF)

Trop J Pharm Res, January 2021; 20(1): 57 
The levels of PIGF were strongly and positively correlated with newborn birth weight $(r=0.853$, $\mathrm{P}<0.001)$ and gestational weeks at delivery $(r=0.900, P<0.001)$, while strongly and negatively correlated with MAP at blood sampling $(r=-0.850$, $\mathrm{P}<0.001)$ and proteinuria $(r=-0.879, \quad P<0.001)$ (Table 2).

\section{ROC analysis}

Figure 2 and Figure 3 present the ROC curve analyses. For the discrimination between controls and PE, when using a cut-off of 8.90 $\mathrm{ng} / \mathrm{ml}$ for Sestrin2 led to $95.1 \%$ sensitivity, $90.4 \%$ specificity, and an AUC of 0.979 , and using a cut-off of $122.50 \mathrm{pg} / \mathrm{ml}$ for PIGF yielded $80.5 \%$ sensitivity, $100 \%$ specificity, and an AUC of 0.963 .

For the discrimination between mild and severe $\mathrm{PE}$, when using a cut-off of $11.15 \mathrm{ng} / \mathrm{ml}$ for Sestrin2 led to $66.7 \%$ sensitivity, $95.7 \%$ specificity, and an AUC of 0.857 , and using a cut-off of $68.50 \mathrm{pg} / \mathrm{ml}$ for PIGF yielded $55.6 \%$ sensitivity, $100 \%$ specificity, and an AUC of 0.837 .

\section{DISCUSSION}

There are many studies on the relation of PE with PIGF [15-17], but there are few regarding the association of Sestrin2. Therefore, this study aimed to explore the levels of serum Sestrin2 and PIGF in pregnant women with PE and their correlation with the severity index of the disease. The results suggest that Sestrin2 and PIGF are correlated with the severity of PE. Both Sestrin2 and PIGF had high value for PE diagnosis and severity prediction while the sensitivity of Sestrin2 is higher than PIGF.

Through the analysis of the three groups of pregnant women, it was found that the levels of MAP at blood sampling, sCr, ALT, AST, LDH, proteinuria were higher, and a lighter newborn birth weight, a younger gestational age at delivery, and a shorter duration between blood sampling and delivery in patients with mild PE or severe PE than that of healthy pregnant women. The above symptoms were more severe in serious PE than in mild PE, as supported by a study by Li et al. [19], suggesting that PE can cause many adverse patient and delivery outcomes with the aggravation of the disease.

The basic pathological changes in PE are the differentiation of maternal placental trophoblasts and abnormal remodeling of spiral arterioles, which lead to the decrease of uterine and placental blood flow [2-5]. Long-term hypoxia will lead to mitochondrial dysfunction and increased reactive oxygen species (ROS) production [20], which will imbalance the antioxidant defense mechanisms. Subsequently, the increase of ROS, endoplasmic reticulum stress (ERS), and placental oxidative stress [21] result in multiple organ injury to the mother and infant and threatening the life of the mother and infant in serious cases. The regeneration effect of PIGF on small blood vessels has been confirmed [810], as well as the antioxidant effect of Sestrin2 and its potential effect in immune-inflammatory reaction $[11,12]$.

At present, multiple studies have shown that the expression of Sestrin2 is increased in hypoxia and oxidative stress [22-24], which can significantly reduce the content of ROS and various inflammatory factors in cells, thus protecting the cells from the effects of oxidative stress and ischemia. Jegal et al. [25] showed that the up-regulation of Sestrin2 expression could inhibit mitochondrial damage and related apoptosis, which is consistent with the results of this study. The ischemic and anoxic environment of PE pregnant women will lead to placental ERS, which will affect physiological processes such as energy metabolism and protein synthesis, modification, and transport [26]. Once ERS exceeds the range of the body's selfregulation ability, it will cause cell apoptosis. Ye et al. [27] found that ERS can induce the expression of Sestrin2 and alleviate the damage of diseases caused by ERS. Those results are supported by those by Tayyar et al. [13], who showed that Sestrin2 is involved in PE, particularly in severe PE. Furthermore, the severity of PE is related to the antioxidant level [20], suggesting that the study of Sestrin2 might provide observation indicators for the early prediction of severe PE, as supported by Tayyar et al. [13].

During the development of PE, placental neovascularization and vasodilation function are closely related to the disease [28]. Related angiogenic factors play an important role. PIGF can maintain the integrity and permeability of normal vascular inner wall structure, provide sufficient nutrients for infants, promote neovascularization, improve blood supply, and relieve the ischemia and hypoxia state of severe PE. PIGF can promote the differentiation of placental trophoblasts, enhance their invasiveness, reduce the apoptosis of trophoblasts, effectively promote the recasting of uterine spiral arteries, and improve the blood supply [29]. 
Soluble fms-like tyrosine kinase-1 (sFlt-1), as a common anti-angiogenic factor, can aggravate the progression of $\mathrm{PE}$ and inhibit the binding of PIGF to the vascular endothelial growth factor receptor. Guo et al. [30] found that Sestrin2 can inhibit the effect of sFlt-1 on antagonizing angiogenesis and activate adenosine phosphatedependent protein kinase (AMPK), while AMPK may activate and increase VEGF, promote neovascularization, and improve the condition of patients. Sestrin2 can induce the expression of nitric oxide synthase, increase the production of $\mathrm{NO}$, and improve the vasodilation function of blood vessels, thus protecting the cells from ischemia [31]. Therefore, the Sestrin2 levels of the pregnant women in the three groups increased with the increase of PE severity, and PIGF levels decreased in turn.

The correlation analysis showed that the levels of Sestrin2 and PIGF were each significantly correlated with various biomarkers of the health status and PE severity, suggesting that Sestrin2 may participate in the occurrence and development of $\mathrm{PE}$, and its level has a certain reference value for judging the severity of $P E$. The ROC curve showed that both Sestrin2 and PIGF had high value for PE diagnosis and severity prediction. Relevant studies showed that the severity of PE is related to antioxidant status [32]. The increase of the Sestrin2 levels reflects the oxidative stress level of pregnant women with severe PE, and Sestrin2 could inhibit the antagonistic effect of sFlt-1 on blood vessels and promote PIGF to play its role against PE. Therefore, Sestrin2 has higher sensitivity. This result suggests the importance of Sestrin2 levels in PE diagnosis and severity judgment.

This study has limitations. PE is a relatively common condition in pregnant women, and the sample size of the present study was relatively small. In addition, all participants were from a single hospital covering a limited geographical area. The results should be validated in larger populations and other ethnicities. Because of the retrospective nature of the study, the correlation analyses were limited to the data available in the charts.

\section{CONCLUSION}

Sestrin2 and PIGF are significantly correlated with the severity of $P E$ and with various biomarkers of the health status and PE severity. The sensitivity of Sestrin2 is higher than PIGF's for $P E$ diagnosis and severity prediction. These results suggest that Sestrin2 could be a novel biomarker for the screening, diagnosis, and management of PE. Future studies could look into whether the development of PE can be prevented by increasing the concentration of Sestrin2.

\section{DECLARATIONS}

\section{Acknowledgement}

None provided.

\section{Conflicts of interest}

No conflict of interest is associated with this work.

\section{Contribution of authors}

We declare that this work was done by the authors named in this article and all liabilities pertaining to claims relating to the content of this article will be borne by the authors.

\section{Open Access}

This is an Open Access article that uses a funding model which does not charge readers or their institutions for access and distributed under the terms of the Creative Commons Attribution License (http://creativecommons.org/licenses/by/ 4.0) and the Budapest Open Access Initiative (http://www.budapestopenaccessinitiative.org/rea d), which permit unrestricted use, distribution, and reproduction in any medium, provided the original work is properly credited.

\section{REFERENCES}

1. Duley L. The global impact of pre-eclampsia and eclampsia. Semin Perinatol. 2009;33(3):130-7. Epub 2009/05/26. doi: 10.1053/j.semperi.2009.02.010. PubMed PMID: 19464502.

2. Leeman L, Dresang LT, Fontaine $P$. Hypertensive Disorders of Pregnancy. American family physician. 2016;93(2):121-7. PubMed PMID: 26926408.

3. Magee LA, Pels A, Helewa M, Rey E, von Dadelszen $P$, Canadian Hypertensive Disorders of Pregnancy Working G. Diagnosis, evaluation, and management of the hypertensive disorders of pregnancy: executive summary. Journal of obstetrics and gynaecology Canada : JOGC = Journal d'obstetrique et gynecologie du Canada : JOGC. 2014;36(5):416-41. doi: 10.1016/s1701-2163(15)30588-0. PubMed PMID: 24927294.

4. Regitz-Zagrosek V, Roos-Hesselink JW, Bauersachs J, Blomstrom-Lundqvist C, Cifkova R, De Bonis $M$, et al. 2018 ESC Guidelines for the management of cardiovascular diseases during pregnancy. European heart journal. 2018;39(34):3165-241. doi: 10.1093/eurheartj/ehy340. PubMed PMID: 30165544.

5. ACOG Practice Bulletin No. 202: Gestational Hypertension and Preeclampsia. Obstetrics and gynecology. 2019;133(1):e1-e25. doi: 10.1097/AOG. 0000000000003018 . PubMed PMID: 30575675.

6. Report of the National High Blood Pressure Education 
Program Working Group on High Blood Pressure in Pregnancy. American journal of obstetrics and gynecology. 2000;183(1):S1-S22.

7. Cai L, Liu J, Wu S, Zhu B. Clinical Value of Soluble FmsLike Tyrosine Kinase-1/Placental Growth Factor and Uterine Artery Pulsatility Index in Predicting Preeclampsia. Chin Gen Pract. 2018;21(7):827-30.

8. Iwasaki $H$, Kawamoto A, Tjwa M, Horii M, Hayashi $S$, Oyamada A, et al. PIGF repairs myocardial ischemia through mechanisms of angiogenesis, cardioprotection and recruitment of myo-angiogenic competent marrow progenitors. PLoS One. 2011;6(9):e24872. Epub 2011/10/05. doi: 10.1371/journal.pone.0024872. PubMed PMID: 21969865; PubMed Central PMCID: PMCPMC3182165.

9. Zhang $H$, van Olden $C$, Sweeney $D$, Martin-Rendon $E$. Blood vessel repair and regeneration in the ischaemic heart. Open Heart. 2014;1(1):e000016. Epub 2014/10/22. doi: 10.1136/openhrt-2013-000016. PubMed PMID: 25332783;

10. Eelen G, Treps L, Li X, Carmeliet P. Basic and Therapeutic Aspects of Angiogenesis Updated. Circ Res. 2020;127(2):310-29. Epub 2020/08/25. doi: 10.1161/CIRCRESAHA.120.316851. PubMed PMID: 32833569 .

11. Yi W, Zhang Y, Guo Y, Li D, Li X. Elevation of Sestrin-2 expression attenuates Sevoflurane induced neurotoxicity. Metab Brain Dis. 2015;30(5):1161-6. Epub 2015/05/15. doi: 10.1007/s11011-015-9673-1. PubMed PMID: 25971460.

12. Dong $B$, Xue $R$, Sun $Y$, Dong $Y$, Liu C. Sestrin 2 attenuates neonatal rat cardiomyocyte hypertrophy induced by phenylephrine via inhibiting ERK1/2. Mol Cell Biochem. 2017;433(1-2):113-23. Epub 2017/05/13. doi: 10.1007/s11010-017-3020-2.

13. Tayyar AT, Tayyar A, Kozali S, Karakus R, Eser A, Abide Yayla $C$, et al. Maternal serum sestrin 2 levels in preeclampsia and their relationship with the severity of the disease. Hypertension in pregnancy. 2019;38(1):13-9

14. Wang LX, Zhu XM, Yao YM. Sestrin2: Its Potential Role and Regulatory Mechanism in Host Immune Response in Diseases. Frontiers in immunology. 2019;10:2797. doi: 10.3389/fimmu.2019.02797.

15. Chau K, Hennessy A, Makris A. Placental growth factor and pre-eclampsia. Journal of human hypertension. 2017;31(12):782-6. doi: 10.1038/jhh.2017.61. PubMed PMID: 29115294.

16. Agrawal S, Shinar S, Cerdeira AS, Redman C, Vatish M. Predictive Performance of PIGF (Placental Growth Factor) for Screening Preeclampsia in Asymptomatic Women: A Systematic Review and Meta-Analysis. Hypertension. 2019;74(5):1124-35.

17. Duhig KE, Myers J, Seed PT, Sparkes J, Lowe J, Hunter $R M$, et al. Placental growth factor testing to assess women with suspected pre-eclampsia: a multicentre, pragmatic, stepped-wedge cluster-randomised controlled trial. Lancet. 2019;393(10183):1807-18. doi: 10.1016/S0140-6736(18)33212-4.

18. Yang Z, Zhang W. Interpretation of Guidelines for Diagnosis and Treatment of Hypertensive Disorder Complicating Pregnancy. Chin J Pract Gynecol Obst. 2015;31(10):886-93.

19. Li X, Xai J, Gan Y. Related Influencing Factors and Maternal and Infant Outcomes of Low Birth Weight Infants in Preeclampsia Pregnant Women. Matern
Child Health Care China. 2019;34(6):1252-5.

20. Wang $C$, Chang $R$, Huang $X$. Significance of Serum Vascular Factors and Oxidative Stress Status in Patients with Severe Preeclampsia. Hebei Med J. 2018;40(11):1613-6, 20.

21. O'Brien M, Baczyk D, Kingdom JC. Endothelial Dysfunction in Severe Preeclampsia is Mediated by Soluble Factors, Rather than Extracellular Vesicles. Sci Rep. 2017:7(1):5887. Epub 2017/07/21. doi: 10.1038/s41598-017-06178-z.

22. Guo M, Xia Z. Research Progress of Antioxidant Stress Effect of Sestrin2 on Ischemia-Reperfusion. China Med Herald. 2017;14(17):28-31.

23. Chuang YC, Yang JL, Yang DI, Lin TK, Liou CW, Chen SD. Roles of Sestrin2 and Ribosomal Protein S6 in Transient Global Ischemia-Induced Hippocampal Neuronal Injury. Int J Mol Sci. 2015;16(11):26406-16. Epub 2015/11/12. doi: 10.3390/ijms161125963. PubMed PMID: 26556340.

24. Zeng YC, Chi F, Xing R, Zeng J, Gao S, Chen JJ, et al. Sestrin2 protects the myocardium against radiationinduced damage. Radiat Environ Biophys. 2016;55(2):195-202. Epub 2016/03/17. doi: 10.1007/s00411-016-0643-8.

25. Jegal KH, Ko HL, Park SM, Byun SH, Kang KW, Cho IJ, et al. Eupatilin induces Sestrin2-dependent autophagy to prevent oxidative stress. Apoptosis. 2016;21(5):642 56. Epub 2016/03/27. doi: 10.1007/s10495-016-12336. PubMed PMID: 27015669.

26. Sundararajan S, Jayachandran I, Balasubramanyam $M$, Mohan V, Venkatesan B, Manickam N. Sestrin2 regulates monocyte activation through AMPK-mTOR nexus under high-glucose and dyslipidemic conditions. J Cell Biochem. 2018. Epub 2018/11/20. doi: 10.1002/jcb.28102. PubMed PMID: 30450765

27. Ye J, Palm W, Peng M, King B, Lindsten T, Li MO, et al. GCN2 sustains mTORC1 suppression upon amino acid deprivation by inducing Sestrin2. Genes Dev. 2015;29(22):2331-6. Epub 2015/11/07. doi: 10.1101/gad.269324.115. PubMed PMID: 26543160.

28. Zhang Z, Song W, Zhang L. Research Progress on the Mechanism of Expression Changes of Angiogenic Factors and Anti-angiogenic Factors in the Pathogenesis of Preeclampsia. Shandong Med J. 2017;57(15):112-4.

29. Makris A, Yeung KR, Lim SM, Sunderland N, Heffernan $S$, Thompson JF, et al. Placental Growth Factor Reduces Blood Pressure in a Uteroplacental Ischemia Model of Preeclampsia in Nonhuman Primates. Hypertension. 2016;67(6):1263-72. Epub 2016/04/20. doi: 10.1161/HYPERTENSIONAHA.116.07286.

30. Guo M, Zhao B, Lei S. The Role of Autophagy Mediated by Sestrin2/Adenosine Monophosphate Activated Protein Kinase in Myocardial Ischemia-Reperfusion Injury in Diabetic Rats and the Protective Effect of TertButylhydroquinone Preconditioning. Chin J Diab Mell. 2017;9(6):382-7.

31. Wang X, He J, Zhao Q. The Protective Effect and Mechanism of Sestrin2 Overexpression on HypoxiaReoxygenation Injury of Rat Hippocampal Neurons. Chin J Neuromed. 2019;18(8):779-84.

32. Zhang J, Wang L, Zhang X. Correlation Analysis of Serum Sestrin2 Level with Severity and Prognosis in Patients with Acute Ischemic Stroke. Hebei Med. 2019;25(2):259-62. 